\title{
Las infancias indígenas como configuración diferencial de las concepciones de infancia
}

Indigenous Childhoods as a Differential Configuration of the Conceptions of Contemporary Childhood

A criança indígena como configuração diferencial das concepções de infância contemporâneo

Javier Alfredo Fayad-Sierra * (iD https://orcid.org/0000-0002-5414-7986

\section{Para citar este artículo}

Fayad-Sierra, J. A. (2021). Las infancias indígenas como configuración diferencial de las concepciones de infancia. Revista Colombiana de Educación, 1(83), 1-20. https://doi.org/10.17227/rce.num83-11900.

Fecha de recepción: 12/06/2020

\section{(9) (1) @ 8}




\section{Resumen}

Palabras clave: infancias; infancias indígenas; ciclos de vida

Keywords: childhoods; indigenous childhoods; life cycles

Palavras-chave: infância; infância indígena; ciclos de vida
Existen diferencias culturales en la concepción de infancia entre el modo de concebir las familias y la educación desde lo global y lo cultural particular de las comunidades indígenas, son lógicas diferentes porque una le entregan los cuidados a instituciones especializadas y la otra requiere conservar ciertas tradiciones de cuidados que aseguren la pervivencia como pueblos diferenciales; una sistematiza y aplica y la otra realiza experiencias y vivencias que siguen el ciclo de vida y las pautas de crianza de la cultura particular. Las infancias en la contemporaneidad son cada vez más mecanizadas y encerradas, se distancian de sus esencias culturales y de sus influencias étnicas que son productoras de sentidos culturales especiales. Parece ser que las infancias se quedan en las influencias de la comunicación y consumos que reproducen ciertas formas institucionales que aleja a las generaciones de la influencia de pautas de crianza de la cultura. En el caso de los pueblos indígenas en Colombia, hay riquezas en estas formas de cuidar y transmitir la cultura desde las cosmovisiones, tradiciones culturales, quienes se encuentran en una situación de cómo los modos de institucionalización de las infancias, desde entidades como el Instituto Colombiano de Bienestar Familiar, desconoce la importancia de estas formas culturales y produce una crisis de representación, valoración y visibilidad de las infancias de los pueblos indígenas.

\section{Abstract}

There are cultural differences in the conception of childhood between the way of conceiving families and education from the global and the particular cultural of indigenous communities, they are different logics because one gives care to specialized institutions and the other requires the preservation of certain traditions of care that ensures survival as differential peoples; one systematizes and applies and the other carries out experiences and experiences that follow the life cycle and upbringing guidelines of the particular culture. Today's childhoods are increasingly mechanized and enclosed, distancing them from their cultural essences and from their ethnic influences that are producers of special cultural meanings. It seems that childhoods remain in the influences of communication and consumption that reproduce certain institutional forms that move generations away from the influence of cultural upbringing patterns. In the case of indigenous peoples in Colombia, there are riches in these ways of caring for and transmitting culture from worldviews, cultural traditions, who are in a situation of how to institutionalize childhood from entities such as the Colombian Institute of Family Welfare it ignores the importance of these cultural forms, producing a crisis of representation, appreciation and visibility of the childhoods of indigenous peoples.

\section{Resumo}

Existem diferenças culturais na concepção de infância entre a forma de conceber as famílias e a educação a partir do global e da cultura particular das comunidades indígenas, são lógicas distintas porque uma atende instituições especializadas e a outra exige a preservação de certas tradições de cuidado que garante a sobrevivência como povos diferenciais; um sistematiza e aplica e o outro realiza experiências e vivências que acompanham o ciclo de vida e as diretrizes de formação de uma cultura particular. As infâncias de hoje são cada vez mais mecanizadas e fechadas, distanciando-as de suas essências culturais e de suas influências étnicas que são produtoras de significados culturais especiais. Parece que a infância permanece nas influências da comunicação e do consumo que reproduzem certas formas institucionais que afastam gerações da influência dos padrões de educação cultural. No caso dos povos indígenas colombianos, há riquezas nessas formas de cuidar e transmitir cultura a partir de visões de mundo, tradições culturais, que se encontram em situação de institucionalizar as infâncias de entidades como o Instituto Colombiano de Bem-Estar Familiar ignora a importância dessas formas culturais, produzindo uma crise de representação, valorização e visibilidade das infâncias dos povos indígenas. 


\section{Introducción}

Este artículo es una reflexión sobre un aspecto con poca difusión: la educación de las infancias indígenas desde algunas concepciones tradicionales indígenas. Nos proponemos retomar algunos elementos sobre las infancias indígenas y sus ciclos de vida como caracterización de la cultura, de algunos elementos de las prácticas culturales, que presentamos como parte de varias experiencias de acompañamiento en procesos educativos en comunidades Nasa y Misak, en el departamento del Cauca, además de la realización del diplomado sobre Educación de la Primera Infancia en Diversidad Cultural, realizado por la Universidad del Valle en el segundo semestre del 2016, con líderes, agentes educativos y representantes de los Cabildos Indígenas de la ciudad de Cali.

Consideramos que existe una diferencia sustancial en el cómo se conciben las infancias, entre la generalidad global que promueve concepciones evolutivas del desarrollo, que se queda en aspectos del crecimiento, pasos por etapas o edades precisas, y las formas de concebir las infancias en las culturas indígenas, que se centra en los ciclos de vida de la cultura, en la que cada etapa del ciclo está relacionado con el actuar permanente y presente de todos los otros componentes del ciclo de vida, y que muestra articulaciones y relaciones entre cada ciclo con los demás.

Las concepciones de infancia y educación en las tradiciones indígenas en Colombia representan su concepción de vida, se refieren a las formas culturales de cada pueblo que tiene su origen en las cosmovisiones, que se complementa con el uso del idioma y su sentido cultural que está en relación con la naturaleza. De acuerdo con esta visión, todo está vinculado con lo que se conoce como espíritus, que acompañan cada momento y etapa del ciclo de vida, son energías vitales que pertenecen a las opciones de vida en cada uno de los tres mundos en los que conviven las tradiciones indígenas ${ }^{1}$. En este texto se quiere mostrar algunos elementos de las prácticas como las comunidades indígenas del Cauca y de Cali conservan su forma de ver los procesos educativos y formativos en la infancia por medio de la enseñanza de la cultura, sus visiones de mundo y sus prácticas culturales.

En primera instancia, problematizamos las concepciones de reconocimiento y surgimiento de las infancias en la modernidad, retomando autores reconocidos de historia de las infancias y su valoración: Phillippe Ariés (1987), Francoise Dolto (1996), Linda Pollock (1993), Buenaventura Delgado (2000), José Sánchez Parga (2004) y María Victoria Álzate (2003). En un segundo momento, tomamos aportes de las infancias indígenas con los aportes de autores como Francois Correa Rubio (2010), Maritza Díaz y Mauricio Caviedes (2015), Maritza Díaz y Fabián Molina Murillo (2011), Isadora Cruz (2018), Bárbara Muelas (2015), Cabildo Misak (2016), Cabildos Indígenas de Cali (2016).

Estas referencias nos permiten rastrear varias concepciones sobre la valoración de las infancias, por ejemplo, la mirada adultocéntrica sobre las infancias, la psicologización sobre las infancias, la artificialidad contemporánea del rol infantil mediado por las comunicaciones y

1 En las tradiciones andinas y amazónicas aparece esta concepción de relaciones entre el mundo de arriba, que es el mundo de los astros, del cosmos, que influye en lo que pasa en los otros mundos; el mundo de abajo, que es la relación con los ancestros que viven en este espacio espiritual donde mantienen contacto con los otros mundos; el mundo del territorio, que se habita donde las personas conviven y sus prácticas y conocimientos están vinculados con las energías de los otros mundos. 
consumos que producen representaciones mediáticas y la existencia de pautas de crianza diferenciales desde las culturas diversas, otras, de los pueblos indígenas.

Los argumentos generales sobre las infancias son respuesta a la promoción de políticas sobre las infancias, generadas en los cambios de la política pública hacia las infancias a partir de la Convención Internacional sobre los Derechos de los Niños de 1989, la Constitución de 1991, la Ley 12 de 1991, la Ley 1098 del 2006, conocida como Código de la Infancia y la Adolescencia y su reforma en la Ley 1878 de 2018. Estas visiones globales recrean reconocimientos, derechos y deberes de las infancias que son reconocidas como sujetos de derechos. En Colombia, esta concepción globalizada provoca reacciones en las formas como "otras" tradiciones valoran sus infancias, esto porque en las políticas aprobadas sobre los pueblos indígenas desde la Constitución de 1991 se amplían reconocimientos, pero al mismo tiempo se viene enfatizando en globalizar el sentido de la política, en el caso de las infancias porque desde las instituciones encargadas de las infancias como el Instituto Colombiano de Bienestar Familiar (ICBF), se presionan cambios culturales con respecto a la concepción de infancia.

El sentido de retomar y pensar los aportes culturales se hace gracias a la valoración de reconocimientos por medio de normativas especiales, que es lo que ocurre con los pueblos indígenas en las normativas como la Ley 21 de 1991, el Decreto 804 de 1995, la Ley de Lenguas Nativas 1381 de 2010 y el Decreto 1953 de 2014, esta última presenta la política sobre Semillas de Vida ${ }^{2}$, que es como se nombra desde las organizaciones indígenas la Política de Primera Infancia.

En las consideraciones como culturas diferenciales, se asume que hay elementos sobre los cuidados y concepciones sobre las infancias que son particulares a las culturas, algunas de estas visiones las tomaremos de experiencias en contexto nasa y misak del Cauca y aportes del diplomado realizado en el 2016 por la Universidad del Valle con los cabildos nasa, yanakuna, inga y quichua de la ciudad de Cali, en el que participaron algunos orientadores del Centro de Desarrollo Infantil (CDI), de las unidades de atención propias de las comunidades indígenas en Cali, como son los CDI Kushi Tuparina y Wuawua Kuna Pawassi. Este proceso se realizó antes de la configuración del Nido Colibrí, donde se atiende a las infancias indígenas en $\mathrm{Cali}^{3}$.

\section{Las concepciones de infancia desde la mirada global}

En las reflexiones de la historia de la infancia y la niñez, se considera que se trata de edades de la vida que se reconocen recientemente, como dice Ariès, se trataba de una edad de riesgo y de pérdida, no se les reconocía porque no se quería tener apegos ante la mortandad tan alta y se les trataba más como una mascota, se les consideraba dependientes de los adultos, quienes veían la infancia y la niñez como una edad de castigos necesarios y de juegos (Ariès, 1987; Dolto, 1996). Desde la Edad Media, la infancia se caracteriza como una forma de culpar

2 El nombre Semillas de Vida es asignado por algunos pueblos, como los nasa, a sus programas de primera infancia, es un nombre retomado por la propuesta del Sistema de Educación Indígena Propio (SEIP) en el marco de la propuesta de administración e implementación de la educación propia de los pueblos indígenas.

3 Nido es la palabra que en Cali se definió para los denominados centros de desarrollo infantil, se toma esta metáfora del nido como ese lugar que asegura el crecimiento y preparación de los pequeños hasta que puedan defenderse por sí mismos. El Nido Colibrí se crea en octubre del 2017, con una capacidad para atender 300 niños y niñas, donde la mayoría son de las comunidades indígenas de los 6 Cabildos Indígenas existentes en Cali. En este espacio se implementa el programa Semillas de Vida, que es la propuesta de la política de primera infancia indígena en el Decreto 1953 de 2014. 
y negar, asumiendo preceptos religiosos, por eso, cierta lógica histórica plantea que la infancia surge con la modernidad con la razón y el bienestar que implica el cuidado, la higiene y la ciudadanía (Ariès, 1987; Pollock, 1993; Dolto, 1996; Delgado, 2000).

La ilustración y la perspectiva social de los Estados modifican las anteriores miradas sobre la infancia, se valora la influencia del adulto en la infancia como consecuencia del naturalismo y la visión de bondad. Esta imagen se reproduce durante el siglo xx hasta que surge cierta visión de autonomía y construcción de subjetividad del mismo niño a finales del siglo xx (Dolto, 1996). Históricamente, la caracterización de los cuidados y la protección de la infancia se hace reproduciendo las preocupaciones de las madres y padres por sus hijos en los momentos del nacimiento, la alimentación, el destete, la dentición, el llanto, el andar, el hablar, el dormir, la vacunación, como evidencias de interés en los pequeños de esa edad y su proceso de crecimiento (Pollock, 1993).

Dolto (1966) nos dice que en cada niño el ciclo de crecimiento reproduce el ciclo de la humanidad, de alguna manera, cuidar la infancia es reconocer su origen y valorar cierta visión mágica. En estas edades iniciales, es imposible desconocer las influencias de los contextos y abstraer al infante de la influencia de la etnia en la que ha nacido, pero la modernidad, en cuanto concepción de occidente, argumenta este proceso como una razón tecnológica y comunicativa de adaptación al mundo de los adultos. Las infancias crecen sin su propia historia, no saben de su experiencia de paso de una edad a otra en el sentido de las culturas (Dolto, 1996).

Es necesario reconocer que cada cultura tiene una manera particular de clasificar las edades de la vida, con momentos o prácticas que caracterizan el paso de una etapa a otra y permiten valorar las diferencias sobre cómo se cuidan y crían las infancias en las culturas distintas del modelo que define la cultura global de influencia eurocéntrica y anglosajona. De acuerdo con estas, el crecimiento en la infancia y la niñez está íntimamente ligado a descripciones e intervenciones en los primeros años de vida, que se explican por medio de teorías de la psicología evolutiva o de la pediatría, mientras que en otras culturas se manejan visiones centradas en sus costumbres desde sus cosmovisiones.

La historia de la infancia se dedica en gran parte a mostrar en qué momento la infancia es una edad reconocida y diferenciada de las prácticas de los adultos, donde se pretende insertar lo más pronto posible a los infantes en las prácticas de los adultos. En esa lógica, surge la escuela como entidad separada de los adultos y especializada en las edades de los niños (Álzate, 2003). En la modernidad, la infancia pasa a ser valorada y atendida desde la creación de instituciones de salud y educación con la intención de quitarles los cuerpos infantiles a las familias se visualiza como higiene y salud pública en ese momento surgen los hospitales maternos infantiles y las clínicas de maternidad (Ariès, 1987; Álzate, 2003).

La educación formal tipo escolarizada se considera que responde a la niñez (mayores de 5 años), mientras que en la infancia (de 0 a 5 años) la educación se visualiza como un aspecto de interés de las familias, las madres y nodrizas o niñeras, en una forma de cuidadores y guarderías, se trata de procesos de cuidados, juegos, actividades lúdicas, siguiendo lo que hoy se denomina como actividades rectoras (relación con el entono, artes, juego, literatura infantil) $)^{4}$.

4 Estas actividades rectoras responden a las normas sobre la educación infantil que se explican en los fundamentos y lineamientos técnicos de primera infancia de la política De Cero a Siempre del Ministerio de Educación Nacional, que se recoge inicialmente en Graciela Fandiño y Yolanda Reyes (2012). 
En las primeras formas de educación, los padres consideraban que a los niños se les debía modelar su carácter en el sentido de formar su voluntad (Pollock, 1993). Es necesario plantear que la educación como tal en instituciones se inicia con la niñez y no con las infancias, la niñez es cuando los niños dejan atrás la infancia, donde se pasa de la preocupación por la salud, la higiene y cuidados ante los riesgos de las enfermedades y la muerte, a una edad donde la disciplina, los juegos y la educación se convierten en el centro del interés de padres, madres, familias e instituciones especializadas (Pollock, 1993).

La escuela como tal se centra en los procesos de definición de un método de enseñanza que se inicia con Juan Luis Vives, Rene Descartes, Juan Amos Comenio, método que logra expresiones más sistematizadas en los jesuitas, jansenistas y protestantes (Delgado, 2000). Comenio dice que la educación comienza con el nacimiento, con la madre como primera maestra, prosigue con la puericia entre los seis y doce años, la adolescencia, la juventud y la academia o la universidad (Delgado, 2000). Delgado nos plantea la influencia de Pestalozzi en Fröbel, siguiendo la influencia roussoniana de las infancias y especialmente de las infancias abandonadas, donde se piensa la educación desde la naturaleza infantil, aquí se vuelve a insistir en esa diferencia entre la educación infantil centrada más en cuidados, que en instrucción que responde más con la niñez (Delgado, 2000).

En la contemporaneidad, se concibe la escuela desde la concepción burguesa del espacio, del encierro, donde se privatiza el área de la vida familiar y social de la infancia, el encierro representa el acomodo de los niños de las clases ricas. Esta opción formativa es muy limitada porque no tiene en cuenta sus propias vivencias, se centra en crear artificialmente espacios de seguridad, los espacios familiares e institucionales, de educación, salud, recreación, donde se crea cierta cultura de "cargarlos" y tecnologizarlos, de "empacarlos", porque se les determina previamente todo, se les lleva a cargas, se cuida y previene todos los riesgos y ya no adquieren experiencias por sí mismos. Ante esta situación, Dolto dice que las infancias hoy son más vulnerables por la falta de experiencia adquirida día tras día (Dolto, 1996).

\section{La educación en la infancia como formas concretas de reconocer las diferencias culturales}

El adulto reproduce una lógica de masificación, científica, médica, corporal, nutricional, mediática de las infancias, que las aísla de las experiencias de las infancias anteriores, de sus padres, abuelos y de su herencia cultural. La infancia se desconecta de lo que culturalmente ha sido y entra a formas artificiales de crecimiento. El lenguaje al que se adentra es global, los imaginarios, simbolismos que recibe no son los propios de sus tradiciones de origen, no se enlazan con sus dialectos, idiomas, palabras de hacer y nombrar de la cultura propia del grupo al que pertenece (Dolto, 1996).

Cada cultura tiene una concepción de pautas de crianza y cuidados que se proponen desde la puericultura, la pediatría, la psicología y la pedagogía, conciben una infancia y una niñez universal y global, donde se asegura su vida y crecimiento desde los cuidados médicos para disminuir posibles riesgos, se le trata por medio de cuidados e intervenciones a partir del embarazo de la madre, el nacimiento y durante los primeros meses. Esta visión medicalista reemplaza y niega la visión cultural de las cosmovisiones y sus lógicas de cuidados, las infancias son cada vez más intervenidas por el adulto científico, sabio, con las normas y leyes de rendimiento de las técnicas hospitalarias y médicas, donde no se permite ninguna relación 
con la cultura. Todo lo que entra a la clínica o al hospital deja de tener trato con la cultura, todas las energías, flujos, partes como la placenta y el cordón umbilical, que hacen parte de la generación de la vida ahora son tratados como desechos clínicos.

En las culturas indígenas las energías son representadas en los flujos y partes del cuerpo que preparan la vida y tienen un sentido cultural preciso, cualquier expresión de vida está conectada con energías cósmicas, ancestrales y territoriales. Por este motivo es que la placenta, el cordón umbilical, todo lo que representa flujos y conexión entre las dos vidas, madre-bebé, es necesario tener unos cuidados con estos flujos energéticos y por eso se entierran en un lugar simbólico y de equilibrio de flujos de energías, por lo regular dentro de la casa donde se concibe y nace el infante, niño, niña. En forma general, los cuidados hacen parte de las condiciones de las energías vitales en relación directa con la naturaleza, con el contexto, con las fuentes de agua, con la alimentación, que culturalmente son energías que fluyen y afectan a la mujer, por eso hay que tener cuidados previos al embarazo, durante el embarazo, en el parto y posparto y que se continúan con los cuidados en el crecimiento de los infantes, en la locomoción, en el sueño, en el juego ${ }^{5}$.

En las prácticas culturales se valoran ciertas continuidades del crecimiento por medio del conservar y practicar la relación o vínculo con la cultura específica, un ejemplo de esto son las tradiciones de cuidados y acompañamientos que realizan personas expertas de las comunidades, las parteras y médicos tradicionales, quienes actúan como promotores de la relación de pareja, de cuidar la familia, de mantener viva la comunidad. Esto se manifiesta en diferentes formas de preparación de la pareja desde antes de la concepción, en la misma concepción, en el nacimiento y en el crecimiento de la infancia a la niñez y la juventud, que a medida que va creciendo se busca que adquieran niveles de autonomía bajo la mirada de la cultura, del idioma, de sus prácticas que son experiencias concretas.

El conocimiento cultural particular parte de la idea de que hay una concepción de herencia genética, de cosmovisión, de relaciones de energías, de influencias culturales, de sentidos ancestrales, sobre cómo cuidar y acompañar el crecimiento, este saber cultural aporta en una concepción muy particular del reconocimiento de las infancias y en la identidad cultural. Este saber y sus prácticas con sus sabedores o expertos son una evidencia de las diferencias culturales, por ejemplo, cuando se prohíbe el "trabajo infantil" en el mundo urbano tiene sus apreciaciones, mientras que en los contextos indígenas las infancias participan del trabajo, como bebés todo el tiempo están "chumbados" en la espalda de la mamá, donde hay una articulación de energías y complementos entre el cuerpo de la mujer y de los bebés, de tal manera que no hay limitación entre acompañar y participar del trabajo; pero igual el trabajo es un ejemplo formativo para su crecimiento.

En el mundo urbano, el destete y la entrega de los niños y niñas a las guarderías y los hogares infantiles, se insiste en cómo resolver los "miedos", "rupturas", entre la corporalidad de las madres y la corporalidad infantil. En las concepciones urbanas y globales, se considera necesario crear este distanciamiento debido a los tiempos y espacios laborales de las madres

5 La relación madre-bebé es permanente y no se ven como separados, al nacer las energías de la madre siguen afectando al bebé y lo mismo ocurre, del bebé hacia la madre; por eso hay unos cuidados de equilibrar y mantener esa relación por medio de plantas y asistencia de sabedoras, parteras, médicos, que le dan continuidad a las energías existentes en cada momento del proceso de preconcepción, concepción, embarazo, nacimiento.

Número 83, Tercer cuatrimestre de 2021 (septiembre-diciembre) || e-ISSN: 2323-0134 || 7 
y padres, pero en las culturas indígenas estas rupturas no son evidentes porque en las prácticas de trabajo no se necesita crear distancias entre madres e infantes.

También tenemos diferencias en las concepciones de la alimentación, como en los CDI, que reproducen la política del Instituto Colombiano de Bienestar Familiar (ICBF) que operan en los territorios rurales e indígenas, no se permite que se utilicen productos alimenticios del contexto y por lo tanto no se organizan las minutas bajo las concepciones nutricionales de las culturas, pesa más la lógica comercial y contractual que maneja la política administrativa que exige que se contrate con los operadores registrados y aprobados por ellos, donde difícilmente hay operadores del territorio porque no cumplen con los estándares y registros exigidos.

En esta lógica diferencial entre las visiones culturales de las tradiciones indígenas y el mundo urbano global, encontramos el papel del reconocimiento de las sensaciones, sentimientos y percepciones sobre lo simbólico, cultural e imaginarios con que se forma a la infancia. En los CDI hay una sobredimensión de sentidos homogeneizadores de las infancias, los pilares de trabajo o las actividades rectoras, los sentidos del juego, la literatura, los cuentos no se hacen desde los contextos culturales, en el arte se reproducen concepciones urbanas del sentimiento, sensación y percepción de las infancias, desconociendo las formas diferenciales. Las concepciones psicosociales se centran en visiones urbanas, globales donde difícilmente valoran esos otros sentidos, sentimientos, percepciones de las culturas ancestrales.

\section{Las infancias indígenas como expresión de las diferencias culturales}

La pregunta por la representación, la imagen y el imaginario sobre las infancias en lo global, visto desde autores como Ariés (1987), Pollock (1993), Delgado (2000), Sánchez Parga (2003), Álzate (2004), hacen evidente cómo se visualiza históricamente el énfasis de lo angelical, emocional, el arquetipo de pureza, inocencia, castidad y bondad, que produce un sentido idealizado de una etapa de la vida. La concepción global de las infancias se centra en su definición como sujetos de derechos y las políticas de protección de las infancias impulsado por la Unesco, que se reconoce como salud y protección biopsicosocial (Cousiño y Foxley, 2011). Consideramos que las diferencias entre las formas globales de las infancias y las infancias indígenas se centran en la permanencia o cambio en las concepciones de las filiaciones o vínculos familiares y sociales, que cambian según los contextos culturales y sus prácticas de cuidados y protección desde los padres, madres y familias.

En el contexto urbano las filiaciones se diluyen cada vez más, mientras que en las sociedades rurales, y en especial en las visiones indígenas, el sistema de filiaciones permanece, porque se conservan vínculos comunitarios y familiares que sustentan las representaciones culturales, donde la relación madre-infante-familia se explica desde las tradiciones de la cultura.

Sánchez Parga plantea que el tipo de relación filial es determinante en los cuidados a las infancias, lo filial son las expresiones de pertenencia, cantidad, intensidad y diversidad de las relaciones sociofamiliares con que cuenta el infante. Esta relación filial crea interdependencias que producen prácticas de todo tipo, afectivas, educativas, que hacen que las infancias tengan un modo particular de identidad como grupo, en lo personal y subjetivo (Sánchez, 2004, p.

Número 83, Tercer cuatrimestre de 2021 (septiembre-diciembre) || e-ISSN: 2323-0134 || 8 
35). Las influencias del medio donde crecen las infancias son determinantes en la producción de sus identidades y rasgos simbólicos, que materializan sus representaciones como infancia y en concreto hacen visible el "vínculo" que la sociedad y la familia establecen con los infantes para recrear prácticas de dependencia y protección propias de su contexto sociocultural que define el ser infantil.

En lo contemporáneo, una forma de visualizar la crisis de reconocimiento de las infancias se debe a la pérdida de las filiaciones, por ejemplo, el paso de la familia extensa a la familia nuclear produce altos niveles de orfandades. Sánchez (2004) dice que para poder compensar estas orfandades crecientes las infancias se hacen más visibles institucionalmente, se adquieren cada vez más instrumentos de política pública, reciben más atención jurídica, médica, policial, educativa, rehabilitadora, publicitaria, comercial, callejera, laboral, criminal y penal, produciendo una infancia fracturada e invertebrada, porque se convierte en el sector social con mayor financiamiento humanitario y por consiguiente rentable para muchos intereses y organismos (Sánchez, 2004).

En la concepción de infancia institucional se considera que la educación inicial es un proceso que facilita la educación formal, en el sentido de que desde las prácticas institucionales de educación se logra un buen nivel de aseguramiento de la inclusión social y el desarrollo de la población infantil, especialmente en los grupos más vulnerables. Por ello, puede decirse que su rentabilidad se da en términos económicos y sociales, porque se considera que las intervenciones en la primera infancia ofrecen mayores retornos sociales al compararse con intervenciones en etapas posteriores (Díaz y Molina, 2011). Esta situación plantea cierto debate sobre las valoraciones que desde las culturas se hacen del aporte formativo diferente de la condición natural del crecimiento, que hace que existan diferencias sobre el reconocimiento de las culturas en transformar la dependencia de las infancias de los adultos, al respecto, Correa nos dice:

Recientes estudios consideran que la infancia no puede ser reducida a un periodo de inmadurez que se dirige pasivamente a la integración del mundo de los adultos; la infancia no sólo es tan diversa como las sociedades y culturas, sino que los niños y niñas participan activamente en su construcción, lo que desvirtúa la idea de que la sociedad es una entidad estática que se autorreproduce estructural y funcionalmente. (Correa, 2015, p. 56)

En los pueblos andinos la referencia de sociabilidad o filiaciones, se hace por medio del ciclo de vida, por ejemplo, en algunos pueblos como los Nasa y los Misak del departamento del Cauca, la tradición narra que su origen viene de un bebé que es sacado del agua, que viene con el derrumbe y es sacado para protegerlo y cuidarlo porque representa el origen y la autoridad, que se reconoce como el hijo de los espíritus (csxa'w en nasa yuwe; pishau en namtrik).

En estas tradiciones, las infancias trascienden la concepción evolutiva, biologista de crecimiento, cada cultura tiene sus repertorios de cuidados de las infancias como prácticas que se aprenden y desaprenden.

En los nasa y misak se reconoce que las infancias no parten solo desde el nacimiento, porque los padres y madres, las culturas, las familias son generadoras de unas condiciones energéticas propias del contexto que se habita en la casa grande o territorio cultural, donde las infancias y sus cuidados son acompañados por espíritus especiales de la naturaleza. Se trata de cuidados, comprendidos como estímulos culturales que afectan y recrean opciones sobre cómo las infancias, niños y niñas, están, escuchan, ven, hacen, sienten, sueñan, piensan 
siguiendo la influencia del contexto cultural, de las cosmovisiones y creencias que son las que fundan ciertas prácticas diferenciales de cuidados de las infancias.

Los diferentes ritmos y desarrollos desde la idea de singularidades, individualidades, personalidades, no están solamente en los individuos, hacen parte también de las culturas, se trata de ritmos distintos porque son formas y concepciones de vida diferentes que visualizamos en relación con las diferentes concepciones del "mí" y del "yo", que un grupo humano particular recrea por medio de practicar sus creencias y formas de concebir la identidad de sí mismo (Mead, 1973). No se trata de la simple valoración biológica y orgánica que parte de estructuras corporales iguales, en el sentido humano, que nos pueden definir las mismas expresiones, pero en estas tradiciones indígenas de culturas diferentes se presenta la viabilidad de aprender en contextos variables, con contenidos y formas particulares.

Estos procesos de reconocimientos ocurren en medio de cierta ambigüedad, porque al mismo tiempo que se reconoce la diferencia cultural, se avanza en la influencia de los avances tecnológicos, comunicativos y comerciales, que necesariamente influye en todas las realidades por su capacidad de producir un ritmo mecanicista, digital, universal con la intención de definir los ritmos de todos. Esta situación hace que estos reconocimientos culturales y diferenciales sean más nominales que reales en la implementación de las políticas públicas de infancias, aunque en los planes de vida y el Decreto 1953 de 2014 se plantea las "semillas de vida" que tiene diferencias gruesas con la visión que implementa el Instituto Colombiano de Bienestar Familiar (ICBF).

En las comunidades indígenas las infancias son protegidas porque hay una convicción de que el ser parte de dicha colectividad que promueve ciertas prácticas de cuidados, remedios y acompañamiento de los mayores y mayoras, que pasan por llevar a la práctica los cuidados y consejos para que no sean perezosos, sean colaboradores, sean activos de las prácticas familiares y comunitarias. Las infancias, niños y niñas participan en las actividades de los adultos, participan de los trabajos de las comunidades a través del juego en las parcelas, participan de las mingas, están en relación con la naturaleza en su contexto. Como dice Libia Tattay:

Los niños y niñas están en relación con los mayores como modelo, para que construyan paulatinamente pensamientos, conceptos y relaciones más armónicas con la naturaleza y que trate de comprender las funciones propias de los elementos significativos que hay allí. (Tattay, 2010, p. 162)

En las tradiciones culturales indígenas hay cuidados y acompañamientos por igual a las madres e infancias por medio de actividades de equilibrio y armonización, desde antes de quedar embarazada, la mujer y el hombre participan por igual en la formación espiritual de las nuevas vidas que van a traer. Durante todo el embarazo, el parto y después del nacimiento, hay cuidados y acompañamientos especiales que hacen los sabedores y parteras, que están todo el tiempo cercanos y pendientes aconsejando y siguiendo la seña, el sentir de la madre, el padre y el infante, se cuidan con dietas especiales a la madre y al bebé, hasta cuando el niño o niña levanta la cabeza, se sienta, gatea.

Las madres son las que están más pendientes de las infancias, niños, niñas a medida que van creciendo, los padres participan dando los consejos que por lo regular se hacen en el fogón. Los sabedores y parteras que acompañan están todo el tiempo consultando a la naturaleza sobre qué cosas se ven, sobre lo que puede llegar a ser y hacer si es niño o niña, según estos sentidos se acompañan con plantas, cuidados, músicas y danzas especiales para cada momento del ciclo de vida. Los niños aprenden observando y escuchando de los padres 
y las niñas de observar y escuchar a las madres, quienes brindan experiencias, sensaciones y consejos sobre su vivencia y saber cultural que representa el mundo de vida que esta direccionado por las cosmovisiones y creencias propias de cada comunidad. Los niños y niñas se desempeñan como miembros activos en la comunidad, con cierta autonomía en su propia vida y contribuyen a la producción de la sociedad y la cultura que los rodea.

Estas formas de cuidar y hacer nos muestran que socioculturalmente una parte importante es lo que la cultura aconseja, dice y de antemano sabe qué hacer y qué cuidados tener, pero también es muy importante la actuación de los niños y niñas, sus voces e intereses, que permiten que a medida que crecen reconocen su experiencia y su capacidad como sujetos, para dar cabida a sus propias expresiones sobre sus derechos como infantes. Esta forma de orientar desde las comunidades desvirtúa el determinismo biológico al que tradicionalmente ha sido sometido la concepción global de la infancia, entre otras aquella visión que presupone que el aprendizaje de los niños y niñas descansa en la imitación y la mera instrucción, que tiende a reducirle a una pasiva incorporación de las infancias, niños y niñas al mundo de los adultos (Correa, 2010).

\section{Configuración de las infancias indígenas desde sus prácticas culturales}

La diferencia principal entre las infancias globales y la infancia indígena tiene que ver con la disimilitud en la concepción e implementación del ciclo de vida de la cultura, que incluye desde los más pequeños hasta los más grandes, como respuesta de pervivencia ante las condiciones creadas por las políticas e intervenciones de lógicas capitalistas, religiosas, de producción y de educación que niegan el estar, actuar, sentir, soñar y pensar propio desde la cosmovisión.

La pérdida de las formas culturales y del ciclo de vida como referente permanente de la cultura tiene que ver con la pérdida de la autonomía territorial, producida históricamente por el despojo, producto de los modelos de colonización territorial, que es evidente con la influencia en los cambios de tipo de relación humana con la madre tierra, que contemporáneamente se concreta en las políticas de intervención con agroquímicos, semillas transgénicas, cultivos ilícitos, pérdida de las fuentes de agua que han perjudicado la capacidad productiva de la tierra. Por ejemplo, las semillas limpias tienen poca presencia territorial, situación que cambia las formas naturales de creación y crecimiento de la cultura porque se genera un fracaso en el papel de cultivar como se hacía milenariamente. Esta realidad de la infertilidad del territorio es causa de la infertilidad de las familias y las "semillas de vida" (infancias), en el sentido de que los cambios en la cultura son influidos por la presencia cada vez más fuerte de los modelos urbanos, el uso de los medios y redes de información en todos los aspectos.

Los contenidos de las propuestas de la política pública en los territorios indígenas normativamente pasan por la capacidad y disposición para que sean las mismas autoridades indígenas quienes promuevan, produzcan, acuerden y administren sus propuestas propias de educación a las infancias, debido a que la forma de concebir y llevar a la práctica son diferentes del modelo de las instituciones nacionales, en este caso el ICBF. Con esto las comunidades no pretenden desconocer las políticas, leyes, normas generales, sino que proponen reorientar estas políticas hacia la realidad social y cultural de las comunidades, basados en la Constitución de 1991, en la Jurisdicción Especial Indígena, que promueve la autoridad y justicia propia, y permite que en los territorios se cuente con mandatos, normas, leyes, reglamentos y proyectos desde las concepciones de aplicación del derecho propio y la cultura. 
En el caso de los aspectos normativos de las infancias, niños y niñas se toma la base jurídica nacional y se aplica según el papel de la autoridad jurídica que representa los cabildos y sus programas especiales de implementación de la política pública nacional desde su particularidad cultural.

El Decreto 1953 de 2014, en sus artículos 41 y 42, especifica los aspectos sobre las infancias, niños y niñas, como "semillas de vida", en sus postulados y estrategias orienta la práctica a realizar desde las autoridades por medio de su entidad particular de manejo de la educación, donde se pretende que se implemente una educación relacional siguiendo el ciclo de vida desde la visión de mundo de la comunidad que no es una mirada fragmentada.

Fruto de los procesos de acompañamiento y diálogo con las diferentes comunidades presentamos seis elementos que sustentan los componentes diferenciales de la educación de las infancias en los territorios indígenas. El primero es el punto de partida de todo lo que se hace, porque es el papel que cumple el idioma propio, de cada pueblo indígena como elemento de formación y relación con la tradición de origen y cultural. La revitalización del idioma inicia con los recién nacidos, es el uso permanente del idioma materno que asegura la existencia de un proyecto educativo en los pueblos indígenas desde la primera infancia hasta los niveles más altos de educación, con la pretensión de transformar la historia de intervención, negación y pérdida de los idiomas, que fue la principal lógica del modelo educativo hasta la Constitución de 1991, la Ley 21 de 1991, la Ley 1381 de 2010, entre otras, que formalizan la importancia del idioma materno como sustento de la pervivencia cultural, al fortalecer el idioma en las infancias se está creando cierta exigencia de hacer lo mismo con las familias.

El segundo elemento de interés es la permanencia de la cultura, que se hace desde el reconocimiento, usos y promoción de los ciclos de vida, la cosmovisión, el origen, los ecosistemas, el idioma, las prácticas culturales donde se incluye en forma "integral" y "relacional" las diferentes etapas de vida partiendo de las infancias, niños y niñas, que requiere de mecanismos concretos de transmitir la cultura, esto significa la posibilidad de implementar los contenidos, tiempos, metodologías y espacios de la educación infantil. Este aspecto representa la materialidad del proceso colectivo, histórico y comunal que fortalece las relaciones materiales y espirituales con el entorno y consigo mismo, de las cuales depende la visión de mundo de cada pueblo.

El tercer elemento es la permanencia de la salud, que se refiere al equilibrio que tenemos con la madre tierra y el buen vivir, que responde a los procesos de transmisión de conocimientos de los sabedores, médicos, hombres y mujeres encargados de los cuidados y consejos que se hacen a las familias a medida que el infante va creciendo, siguiendo la espiral de la vida, acompañado con plantas medicinales, alimenticias y especies animales que protegen la energía vital humana, que en la tradición cultural responde a unas prácticas propias de nutrientes, saberes y sabores, que la cultura tiene como parte de su tradición y se materializa cotidianamente en las prácticas de las familias, los ciclos de semillas y productos de los diferentes pisos térmicos.

El cuarto elemento son los espacios pedagógicos, que valoran y reconocen el sentido de la cultura, parte del ciclo de vida, el ciclo de la naturaleza, los materiales del contexto, las prácticas formativas que se planean teniendo en cuenta los conocimientos culturales que están en el territorio, en el tiempo y el espacio de la cosmovisión y sus relaciones con el aquí y el ahora. Estos espacios implican la implementación formativa de las concepciones y principios que argumentan la educación que las generaciones mayores requieren o visualizan como necesarias en las nuevas generaciones y se concretan en expresiones, consejos, valores, formas de estar, hacer, sentir, soñar, pensar propias de cada cultura. 
El quinto elemento es la forma activa de participación de la comunidad en los cuidados, son diferentes personas, no solamente los sabedores, quienes participan en atender y cuidar a los infantes por medio de diversas lógicas de conocimiento que se concretan en cómo se enseña, cómo se acompaña, cómo se está pendiente del proceso de crecimiento, en diferentes momentos participan expertos y expertas que ayudan, direccionan, recomiendan y practican aportando formas concretas de actividades en músicas, danzas, tejidos, oralidades. En algunos pueblos se cuenta con orientadores o dinamizadores que realizan las actividades directas en los espacios formativos de las infancias que están en los territorios.

El sexto elemento es la formación y capacitación de las personas encargadas de estar y hacer las prácticas formativas con las infancias, niños y niñas, desde una perspectiva propia de cada cultura, que implica un reconocimiento y diálogo con los pilares y actividades rectoras que plantean los lineamientos pedagógicos de la primera infancia del icbf y del men, se trata de reconocer posibilidades relacionales de la cultura que promueven el cuidado de las infancias, que se concretan en la creación, disposición, revitalización y construcción de metodologías, prácticas y materiales de acuerdo con el contexto cultural, siguiendo los planteamientos y experiencias de los mayores y mayoras y de los diferentes sabedores en relación con el proceso de vida de las infancias.

\section{Algunos aspectos de interés de las infancias en los Misak}

En el pueblo Misak, los espacios de cuidados, protección y acompañamiento de las semillas de vida o Numisak son los Ureklee (nido de atención), que representan el Nachak donde se encuentra el Nakuk (fogón), base de la educación propia misak, en el fogón se encuentran los tres pingos, que son las tres piedras que representan un triángulo y es fundamental para el pueblo misak, es allí donde inicia la formación integral, donde los Numisak son acompañados por familias, cuidadores, sabedores propios, parteras, médicos tradicionales, taitas y mamas. Este espacio permite que los Numisak interactúen de manera integral con las familias y la comunidad que cuentan con los principios de vida del ser misak (Cabildo Ancestral Misak. Programa de Educación, 2016, p. 34).

Se cuenta también con el espacio de las "casas del taita Payan" que son espacios que representan la relación ancestral con los shures y shuras desde las prácticas culturales de los tres mundos del misak. Estos son espacios donde se implementa el NuIsuk, o pensamiento propio, que representa la tradición cultural que se recibe desde que inicia cada persona su ciclo de vida, que está presente en el diario vivir del misak, ya que con ellos se logra el Pishintø Waramik que es "vivir en armonía entre nosotros mismos y con todas las formas de vida de la naturaleza" (Cabildo Ancestral Misak. Programa de Educación, 2016, p. 46).

Las etapas del ciclo de vida requieren de una relación estrecha con los astros, cometas, estrellas, nubes, rayos, con las aguas, lagunas, ríos, quebradas, lluvias, los minerales, la flora y fauna, las plantas medicinales, la agricultura, la alimentación, los animales, viviendas, la música, la danza, los tejidos, la oralidad, la educación, la salud, todo son lenguajes naturales que permiten a los Numisak orientar de manera integral los procesos de formación para la vida, en el tiempo y en el espacio, por ello son el camino principal que permite organizar los procesos de atención en la modalidad propia. 


\section{Algunos aspectos de interés de las infancias en los Nasa}

En el pueblo Nasa las "semillas de vida" comienzan con el ciclo de vida que parte de la maduración de la semilla, la siembra de la semilla y la germinación de la semilla. Madurar la semilla es la preparación del hombre y la mujer para el embarazo que implica un conocimiento de la cultura y del sentido de la pareja y el papel de la concepción como tal. Sembrar la semilla representa la vida de un nuevo ser, en este momento del ciclo se realizan los rituales que ayudan que la semilla tome las fuerzas y energías de la Tierra, se despiertan los dones en las semillas desde el vientre de la madre. Se hace necesario que para sembrar la semilla se escuche los consejos de la Luna, para prevenir la debilidad de la semilla y del Sol que regala sus energías, además que ayudan a reconocer y valorar si se quiere concebir un niño o una niña o para que germinen o nazcan bien fuertes (Cruz, 2018).

El útero se considera como una mochila donde se siembra la semilla porque es su tejido, en sus hilos se representan los pasos de la creación de la vida, por eso se asemeja con el vientre de la mujer que es el primer espacio donde comienza a crecer la semilla. En Nasa yuwe se dice dü yaja, 'mochila de parir'. En los hilos de esta mochila se simboliza la construcción del camino de la vida que recorre el niño o niña, los hilos hacen vibrar la semilla dependiendo de la fuerza que heredó la semilla desde el vientre y los huecos o los vacíos en el hilado personifican los problemas que van apareciendo en cada paso, por lo cual deberá reflexionar y volver atrás y repasar su vida, para sortear sus pasos y seguir el camino (Cruz, 2018).

El nacimiento se le llama la germinación de la semilla que comienza con el kiwe thë, que analiza si el nuevo ser podía nacer en casa, el éxito del parto dependía de los cuidados que se habían tenido desde que la mujer era niña. La familia, junto con la partera, preparaba con anticipación las cosas necesarias para la atención del parto en la casa (Cruz, 2018, p. 188). Si hubo buena preparación con las lunas la semilla busca su camino, si se desubica la partera lo acomoda. Cada niño nasa está orientado por las energías de los tiempos y la posición de la Luna y el Sol, esto permite que cada ser sea diferente al otro, en la parte física, espiritual e intelectual (Cruz, 2018, p. 189). Una vez el niño salga a la luz la partera con el kiwe thë, identifica el destino del nuevo nasa, esto se hace de acuerdo con la lectura de la naturaleza y de acuerdo con el sitio donde nace, pues cada sitio tiene espíritus diferentes, por eso recoge energías de los cultivos, la huerta y de los caminos (Cruz, 2018, p. 190).

Al mes de nacido se le hace otra armonización al bebé y a la madre se cuidan con dieta especial y baños para prevenir enfermedades y sanar el útero y la fuerza espiritual, igualmente se crea confianza entre la madre y el bebé en la lactancia. El recién nacido se chumba y tiene también cuidados especiales (Cruz, 2018, p. 191).

\section{Algunos aspectos de interés de las infancias en los cabildos Nasa, Yanacona, Inga y Kichwa en Santiago de Cali}

En esta parte queremos compartir algunos elementos del diplomado realizado con las comunidades y cabildos de los pueblos Yanacona, Nasa, Inga y Quichua durante el segundo semestre del 2016, denominado "Fortalecimiento de la atención integral en educación inicial a niños y niñas de la primera infancia en el municipio de Santiago de Cali", realizado con la Subsecretaría de Bienestar Social del municipio de Santiago de Cali y la Universidad del Valle. Si bien cada pueblo tiene características particulares que los diferencia, queremos presentar aquí algunos elementos importantes a manera de posibles generalidades, que representan las realidades sobre cómo se proponen hacer y pensar desde las comunidades y sus autoridades 
el trabajo hacia las infancias indígenas en el contexto de ciudad con la idea de que el vínculo cultural se conserve.

La identidad es una construcción cultural que, así como se aprende se puede desaprender, en el caso de las "semillas de vida" de los seis cabildos existentes en Santiago de Cali al estar en este territorio distante de su origen se crean nuevas identidades, que es necesario conectarlas con sus identidades indígenas como pueblos originarios, donde la cultura y lo cultural en relación con la naturaleza es una característica de la pervivencia de los pueblos.

Hoy en día hay una confrontación entre la comunitarización y la individualización, que en el sentido de la educación inicial es muy importante la formación en valores comunitarios, sociales de identidad y diversidad, porque desde la educación de los pueblos indígenas se quiere que las prácticas culturales sean construcciones de conocimientos de las culturas, que históricamente sostienen la permanencia y continuidad de cada cultura. Por eso, se necesita que desde la infancia se mantengan y estén activos estos conocimientos y tradiciones culturales, sin importar en qué territorio se encuentran, las prácticas culturales son repertorios de conocimiento que se transmiten de generación en generación y hacen parte de las necesidades de reconocimiento de los sistemas completos de organización y procesamiento de los conocimientos de cada cultura.

En los cabildos y comunidades presentes en Cali, el principal referente de cuidados, protección y acompañamiento a las infancias son las familias, quienes están encargadas de promover la autoridad, la relación con el territorio, la permanente vivencia de la cosmovisión y la práctica del idioma propio. La familia es el lugar de revitalización del idioma y de las prácticas culturales, su relación con la naturaleza se reconoce como "la gran casa" que permite la práctica de lo colectivo comunitario.

En la ciudad se mantiene la pregunta por los espacios y los lugares donde las prácticas formativas con las infancias permitan cultivar el sentido de la cultura, sin negar la realidad de estar en una gran ciudad donde se reciben informaciones distintas, que los separan de su ámbito cultural. Con la intención de crear o continuar con una política pública de atención a las infancias, se propuso desde las autoridades de los cabildos existentes en Cali, la figura del Nido, que en el sentido cosmogónico se trata de un espacio que cuenta con los elementos y argumentos necesarios para la expresión cultural de cada pueblo indígena en la ciudad. El sentir de todas las comunidades indígenas es que este espacio representa una posibilidad de convergencia de las prácticas ancestrales y culturales referidas a la primera infancia, es un espacio donde se explica el sentir y la razón de ser de los lazos familiares, que están presentes en los apellidos como en la comunidad y es necesario que los aportes de cada cabildo, de los agentes educativos que participan en estos espacios formativos estén directamente relacionados con la cultura como una posibilidad de mantener la concepción propia de cada pueblo indígena.

La cosmovisión de cada cultura cuenta con unas referencias especiales sobre el ciclo de vida, de cada una de las etapas en las que cada cultura define prácticas de cuidados, de acompañamientos necesarios, donde siempre están los sabedores encargados de compartir los remedios propios a cada etapa del ciclo, los mayores y mayoras que siguen, aconsejan, enseñan, que saben qué prácticas hay que hacer si es niño o niña, y que permite aconsejar a las madres, padres y familias sobre lo que hay que hacer en cada momento del ciclo de vida. Es necesario que se comprenda como un recorrido de la vida a largo plazo y lo que no se haga en cada momento del ciclo se van a crear repercusiones, enfermedades y debilidades, tanto orgánicas como espirituales. Por ejemplo, en el pueblo yanacona la existencia se manifiesta por momentos de vida, espacios, ciclos, tiempos, no se mira como en occidente por etapas 
fragmentadas, ellos como cultura definen los momentos del ciclo de vida como enamoramiento, preñez, alumbramiento (parir), apa (cargar al bebé en la espalda), caminador, mandadero, minga o minguero (Universidad del Valle, 2016d, p. 10) ${ }^{6}$.

En cada cultura existen prácticas de cuidados a la mujer gestante desde antes del embarazo, durante el embarazo, en el parto y posterior al parto, porque se trata de cuidar la semilla de la vida, que son las condiciones de dar vida en el cuerpo de la mujer en la perspectiva de salud de ella misma. Por ejemplo, en el pueblo Nasa se acostumbra que en los cuidados de la madre y el bebé durante el nacimiento se ubique a la embarazada cerca al fuego, se recomienda que la leña sea roble y se deben colocar los pies de la mamá hacia la dirección detrás del sol. Si la partera ve que el frío está afectando se le brinda aguardiente al çxaw, porque si no el frío va para la partera. Se debe tener plantas calientes como artemisa, ruda y salvia, así como chirrincho, para atacar al frío, estas plantas son calentadas en el fuego para colocarlas en la panza, por lo regular esta práctica se hace para acomodar al bebé en el vientre y prepararlo para recibirlo. Cuando la forma de la barriga es redonda es niña y cuando esta puntuda es niño. Se recomienda dar bebidas calientes a la embarazada, no bañarse con agua fría y utilizar permanentemente el chumbe (Universidad del Valle, 2016b).

En las infancias recién nacidas hay unos cuidados, primero en la relación madre-bebé, en cada cultura se trata de seguir cuidados de protección ante posibles enfermedades, por ejemplo, en el pueblo nasa al nacer se hace la limpieza, que es un ritual que armoniza para quitar el "sucio", que quiere decir limpiar las malas energías o curar alguna enfermedad. Esto va acompañado con la siembra del cordón umbilical en la huerta (para que el niño permanezca en el territorio) y la placenta para evitar el sucio y el refrescamiento (Universidad del Valle, 2016b).

Se hacen prácticas de formación según lo que los sabedores sienten o visualizan sobre lo que puede ser en su vida adulta el recién nacido, entonces, se trata de promover sus cualidades y para esto se utilizan remedios diferentes para situaciones concretas, en cada pueblo según su visión de mundo se conoce de plantas y prácticas que se relacionan con la naturaleza y ayudan a promover estas cualidades que se traen, por ejemplo, en el pueblo inga el remedio para los niños rebeldes es bañarlos con agua hervida de verbena, azotándolos levemente con los ramitos de verbena cada vez que se pongan rebeldes o cuando no acatan la autoridad (Universidad del Valle, 2016a).

En el crecimiento es importante enseñar las simbologías, tejidos, músicas, danzas, artes propias de cada comunidad, por eso es importante que en cada etapa del ciclo de vida se haga pro medio del aprender jugando, aprender haciendo y aprender reconociendo la naturaleza y el territorio. El crecimiento y el estar en la cultura requiere del aprendizaje y uso del idioma propio, de la palabra dulce del idioma que ayuda a reconocer y vivir el sentido humanitario que está en el idioma, en su significado y vínculo con la naturaleza, de los tres mundos (del cosmos, del territorio y de los ancestros). En el idioma está completo el repertorio de identidad y pervivencia como pueblos, por ejemplo, en el pueblo quichua es de suma importancia para la identidad que el wawa (niño y niña), que él-ella esté en contacto con la allpa-mama (madre tierra), la importancia de esta conexión se ve reflejada en la forma de crianza de los wawas, esta conexión fortalece los saberes propios, la importancia en que se mira la propia cultura y el asentamiento de los saberes ancestrales. Prácticas como colgar el ombligo del recién nacido a su cuello y dejar secarlo para guardarlo o sembrar la placenta en la tierra son un símbolo

\footnotetext{
${ }^{6}$ Cada comunidad desde su idioma y sus prácticas dan nombres diferentes a cada momento del ciclo de vida.
} 
propio de una conexión sagrada, la cual cuidará y dará sabiduría al wawa en sus momentos menos favorables (Universidad del Valle, 2016c).

En cada pueblo indígena presente en la ciudad de Cali hay choques y dificultades producidas por no estar materialmente en el territorio, esto se subsana de alguna manera, manteniendo las prácticas de rituales, armonizaciones y medicinas que permiten seguir en contacto con las plantas, las aguas, los territorios y los conocimientos ancestrales. Las prácticas de las medicinas y los rituales de sanación, equilibrio y purificación que tiene cada cultura se hace necesario que se realicen permanentemente en las familias y en las reuniones de la comunidad de cada pueblo indígena en la ciudad.

El aspecto de la alimentación y de las minutas de alimentación en los espacios formativos de las infancias, niños y niñas requiere retomar los conocimientos, prácticas y productos que cada comunidad tiene como acervo cultural. Se trata de recursos energéticos, de saberes que incluyen una relación con los ecosistemas y un sentido de energías que cada persona desde el vientre de la madre y en su crecimiento configura una forma cultural, por ejemplo, como reconocer y valorar que el alimento representa su vida y su relación con las plantas, especies, animales, aguas, que son sabores que identifican su propio universo simbólico y espiritual. El alimento no es solo comer es conservar la cultura y la relación ancestral con el territorio, en este sentido, las comunidades indígenas asentadas en Cali encuentran en el alimento una conexión y presencia con su territorio, al igual que el papel cumple la medicina y las plantas que se utilizan para refrescamientos y protección de energías.

\section{Conclusiones}

La modernidad y la contemporaneidad se convirtió en un referente de pérdida de las diferencias desde las culturas y la valoración del origen étnico, como nos dice Francoise Dolto (1996), cada niño reproduce en su crecimiento el ciclo de la humanidad. Esto nos lleva a hacer la referencia de cómo las culturas definen su camino de la vida desde sus prácticas culturales, cada etapa de la vida significa no solamente crecimiento, desarrollo, porque en cada cultura hay referentes formativos distintos. Esta modernidad y contemporaneidad se ha encargado de crear una figura de infancias institucionalizadas, medicalizadas y asépticas que no guardan memoria cultural y que mantienen en encierros y universos espaciales limitados y controlados.

Las infancias de las "otras" culturas distintas de la global nos plantean formas y concepciones de referencia con la cultura, el medio, lo social desde cosmovisiones diferentes y lógicas otras. Las culturas globalizadas se distanciaron ya hace bastante tiempo de ciertas lógicas naturales, espaciales, humanas que permitían una visión de los cuidados y del crecimiento natural con el entorno, pero desafortunadamente ocurre todo lo contrario, la cultura global se mecaniza y tecnologiza cada vez más su forma de vida. Los cuidados se entregan a modelos de intervención médica, de consumos y encierros sin la participación de las familias y sus saberes.

La pérdida de las filiaciones reproduce instituciones encargadas de cuidar y proteger a las infancias, como plantea Sánchez Parga (2004), pero esta misma institucionalización hace que se pierda el vínculo con la cultura. En las culturas "otras" de los pueblos indígenas se manejan unas concepciones de saberes que retoman aspectos de la naturaleza, de energías y flujos que ayudan a que las infancias permanezcan en relación con sus ancestros, padres y madres, cuenten con saberes de cuidados, protección y acompañamiento que previenen enfermedades y situaciones a futuro.

Se visualiza que existen dos posturas, la de la institucionalización y la de las formas culturales adentro de los pueblos indígenas, que cuentan con mecanismos de cuidados y 
educación de sus infancias que difícilmente se acomodan a las formas de normalización y medicalización de las infancias que plantea la cultura global.

En los pueblos indígenas se cuenta con formas de cuidados que se hacen desde la preconcepción, la concepción y el nacimiento, que organizan el camino de la vida de esa persona que está por nacer. Es decir, hay conocimientos de larga duración que mejoran o cambian situaciones ante posibles riesgos, son conocimientos de protección y prevención, porque no se espera que se presenten las enfermedades para realizar prácticas de cuidado. Mientras que del lado de la globalidad todo queda en manos del servicio de salud y algunos cuidados básicos que se aprenden en las familias o en internet, pero no es preventivo y solo interviene ante la presencia de la enfermedad o del daño.

La valoración filial y espacial del contexto es determinante en la protección de las infancias, son saberes y conocimientos acumulados y trasmitidos en cada cultura a sus expertos, sabedores, médicos y parteras, que, como conocimientos con sus lógicas particulares, reconocen y sustentan el crecimiento de las infancias por medio de ciclos de vida, prácticas culturales que son estímulos activos, que intervienen en la solución de situaciones concretas de la madre, del bebé y de las familias. Estas prácticas se van modificando en la medida que las infancias crecen y hay más autonomía orgánica, biológica y cultural.

Es importante reconocer que existen lógicas, formas concretas y sabedores o expertos en realizar las acciones que en las comunidades se proponen sobre referencias necesarias en la educación y acompañamiento a las infancias que aquí presentamos como síntesis en seis elementos que recogen la importancia y validez de estas prácticas.

\section{Referencias}

Álzate, M. (2003). Infancia: concepciones y perspectivas. Papiro.

Ariès, P. (1987). El niño y la vida familiar en el antiguo régimen. Taurus.

Cabildo Ancestral Misak. (2016). Numisakwan esik uninkatik, nu isuikwan untakteka, kusrennep pera maramik. Formación integral a los Numisak desde el ciclo de vida. Programa de Educación.

Correa, F. (2010). Infancia y trabajo infantil indígena en Colombia. Universidad Nacional de Colombia.

Correa, F. (2010). La formación de la autonomía desde la infancia entre pueblos indígenas colombianos. En F. Correa, Infancia y trabajo infantil indígena en Colombia. Organización Internacional del trabajo (OIT), Universidad Nacional de Colombia.

Cousiño, F. y Foxley, A. (2011). Políticas Públicas para la Infancia. Comisión Nacional Chilena de Cooperación con Unesco.

Cruz, I. (2018). La fuerza del ombligo, pedagogía de la vida. Revista Ciencia e Interculturalidad, 23(2), 174-192.

Delgado, B. (2000). Historia de la infancia. Ariel.

Díaz, M. y Caviedes, M. (2015). Infancia y educación. Análisis desde la antropología. Pontificia Universidad Javeriana. 
Díaz, M. y Molina, F. (2011). Lineamientos pedagógicos para la educación inicial indígena en Bogotá. Alcaldía Mayor de Bogotá, Secretaría Distrital de Integración Social. Organización de Estados Iberoamericanos (OEI), Subdirección para la Infancia.

Dolto, F. (1996). La causa de los niños. Paidós.

Fandiño, G. y Reyes, Y. (2012). Una propuesta pedagógica para la educación de la primera infancia. Documento base para la construcción del lineamiento pedagógico de la educación inicial nacional. MEN.

Mead, H. (1973). Espíritu, persona y sociedad. Desde el punto de vista del conductivismo social. Paidós.

Muelas, B. (2015). өsik uninkatik nu isuikwan kusrennep pera maramik. Educación propia desde el ciclo de vida misak en la primera infancia. Revista Pueblos Indígenas y Educación, (64).

Pollock, L. (1993). Los niños olvidados. Reflexiones entre padres e hijos de 1500 a 1900. Fondo de Cultura Económica.

Sánchez, J. (2004). Orfandades infantiles y adolescentes. Introducción a una sociología de la infancia. Abya Yala.

Tattay, L. (2010). Niños y niñas del Cauca Indígena. En F. Correa, Infancia y trabajo infantil indígena en Colombia. Organización Internacional del trabajo (OIT), Universidad Nacional de Colombia.

Universidad del Valle. (2016a). Experiencias, pautas de crianza y cuidados pueblo inga. Fortalecimiento de la atención integral en educación inicial a niños y niñas de la primera infancia en el municipio de Santiago de Cali.

Universidad del Valle. (2016b). Experiencias, pautas de crianza y cuidados pueblo nasa. Fortalecimiento de la atención integral en educación inicial a niños y niñas de la primera infancia en el municipio de Santiago de Cali.

Universidad del Valle. (2016c). Experiencias, pautas de crianza y cuidados pueblo quichwa. Fortalecimiento de la atención integral en educación inicial a niños y niñas de la primera infancia en el municipio de Santiago de Cali.

Universidad del Valle. (2016d). Experiencias, pautas de crianza y cuidados pueblo yanacona. Fortalecimiento de la atención integral en educación inicial a niños y niñas de la primera infancia en el municipio de Santiago de Cali. 